\title{
Article \\ Dynamic Conditional Bias-Adjusted Carry Cost Rate Futures Hedge Ratios
}

\author{
Dean Leistikow, Yi Tang *(D) and Wei Zhang
}

Gabelli School of Business, Fordham University, 140 West 62nd Street, New York, NY 10023, USA; leistikow@fordham.edu (D.L.); wzhang167@fordham.edu (W.Z.)

* Correspondence: ytang@fordham.edu; Tel.: +1-646-312-8292

Citation: Leistikow, Dean, Yi Tang, and Wei Zhang. 2022. Dynamic Conditional Bias-Adjusted Carry Cost Rate Futures Hedge Ratios. Journal of Risk and Financial Management 15: 12. https://doi.org/ 10.3390/jrfm15010012

Academic Editor:

Shigeyuki Hamori

Received: 23 November 2021

Accepted: 29 December 2021

Published: 3 January 2022

Publisher's Note: MDPI stays neutral with regard to jurisdictional claims in published maps and institutional affiliations.

Copyright: (C) 2022 by the authors. Licensee MDPI, Basel, Switzerland. This article is an open access article distributed under the terms and conditions of the Creative Commons Attribution (CC BY) license (https:// creativecommons.org/licenses/by/ $4.0 /)$.

\begin{abstract}
This paper proposes new dynamic conditional futures hedge ratios and compares their hedging performances along with those of common benchmark hedge ratios across three broad asset classes. Three of the hedge ratios are based on the upward-biased carry cost rate hedge ratio, where each is augmented in a different bias-mitigating way. The carry cost rate hedge ratio augmented with the dynamic conditional correlation between spot and futures price changes generally: (1) provides the highest hedging effectiveness and (2) has a statistically significantly higher hedging effectiveness than the other hedge ratios across assets, sub-periods, and rolling window sizes.
\end{abstract}

Keywords: carry cost rate; hedge ratio; conditional hedge ratio; bias adjustments

JEL Classification: G01; G11; G12; G17

\section{Introduction}

The goal of futures hedging is to reduce the firm's risk and increase its value. A key consideration for a futures hedger is the ratio of futures assets to short over the number of spot assets long, i.e., the futures hedge ratio. Not surprisingly, a large literature has evolved regarding the best hedge ratio estimation technique, i.e., that which is expected to reduce the hedger's risk the most. Wang et al. (2015) empirically tested many hedge ratio estimation methods and found that they fail to beat the naïve hedge ratio.

This paper examines dynamic conditional futures hedge ratios. It focuses on: (1) the economics-based carry-cost rate hedge ratio introduced in Leistikow et al. (2020) and employed in Leistikow and Chen (2019), (2) a hedge ratio based on the Engle (2002) statistics-based dynamic conditional correlation model, and (3) three bias-adjusted versions of the carry-cost rate hedge ratio, one of which incorporates the Engle (2002) dynamic conditional correlation model. It compares the hedge ratios' hedging performances across three broad asset classes to those of each other and those of two common benchmark hedge ratios. The carry-cost rate hedge ratio when augmented with the dynamic conditional correlation between the spot and futures price changes generally (1) provides the highest hedging effectiveness and (2) has a statistically significantly higher hedging effectiveness than either that of the common benchmark hedge ratios or the other approaches across: assets, sub-periods, and rolling window sizes. Moreover, it explains why the carry-cost rate hedge ratio, when augmented with the dynamic conditional correlation between the spot and futures price changes, performs better than the naïve hedge ratio.

Section 2 of the paper discusses the hedge ratios studied. Section 3 specifies the data employed. Section 4 presents statistics regarding the hedge ratios, where the hedge ratios are calculated using a 1008 trading day (i.e., 4 years) rolling window. Alternative rolling window sizes (of 2 and 6 years) are analyzed and found to yield similar results; these results are available in Appendices that will be provided upon request from the authors. Section 5 shows the hedge ratios' hedging performances and relative hedging performances. Section 6 concludes the paper. 


\section{Risk-Minimizing Hedge Ratio Estimation}

For each unit of the spot asset, the hedge ratio $(h)$ for the one-unit futures contract that minimizes the future period's risk is:

$$
h=\frac{\rho \sigma_{s}}{\sigma_{F}}
$$

where: $\sigma_{S}$ is the standard deviation of the spot price change $(\Delta S)^{1}, \sigma_{F}$ is the standard deviation of the futures price change $(\Delta F)$ and $\rho$ is the correlation between $\Delta S$ and $\Delta F$. In this section, we discuss alternative empirical hedge ratio measures.

\subsection{The Traditional Hedge Ratio}

The traditional way to estimate the risk-minimizing hedge ratio on day $d$ is to run the ordinary least square regression:

$$
\Delta S_{d}=a+h_{t} \Delta F_{d}+\epsilon_{d}
$$

where the slope coefficient of $\Delta F, h_{t}$, is the "traditional" hedge ratio. In this study, we estimate Equation (2) on day $d$ using a rolling-window out-of-sample procedure, where $\Delta S_{d}$ and $\Delta F_{d}$ are daily spot and future price changes over the past 1008 trading days (i.e., day d-1007 to day $\mathrm{d}$ ), respectively. ${ }^{2}$ The 1008 -trading days, i.e., 4 -year, rolling window is updated daily. Moreover, we use the same 1008-trading days rolling window when estimating alternative models (discussed below) for the hedge ratio estimated on $d$ to ensure that they are compared on an equal basis. This "traditional" hedge ratio is discussed in mainstream textbooks, e.g., Hull (2018) and has traditionally been used as the benchmark for alternative hedge ratios that have been proposed over the last 40 years.

\subsection{The "Dynamic Conditional" Hedge Ratio}

Equation (2) is often considered a static model in that the historical data are assumed to be equally informative about future realizations. In this subsection, we relax this assumption by estimating an autoregressive-based model that allows more recent observations to be weighted more heavily. Our rationale is that if the dynamics of the underlying variable are indeed time-varying, an appropriately chosen dynamic conditional model is likely to outperform the static model. Engle (2002) proposes a new class of multivariate models called dynamic conditional correlation (DCC) models that are used to predict the time-varying correlations between two financial assets. These models have the flexibility of univariate GARCH models coupled with parsimonious parametric models for the correlations. ${ }^{3}$ Following Engle (2002), we estimate the conditional covariance between $\Delta S$ and $\Delta F$ :

$$
\begin{gathered}
\Delta S_{d}=\alpha_{0}^{\Delta S}+\varepsilon_{\Delta S, d} \\
\Delta F_{d}=\alpha_{0}^{\Delta F}+\varepsilon_{\Delta F, d \prime} \\
E_{d}\left[\varepsilon_{\Delta S, d+1}^{2}\right] \equiv \sigma_{\Delta S, d+1}^{2}=\beta_{0}^{\Delta S}+\beta_{1}^{\Delta S} \varepsilon_{\Delta S, d}^{2}+\beta_{2}^{\Delta S} \sigma_{\Delta S, d}^{2} \\
E_{d}\left[\varepsilon_{\Delta F, d+1}^{2}\right] \equiv \sigma_{\Delta F, d+1}^{2}=\beta_{0}^{\Delta F}+\beta_{1}^{\Delta F} \varepsilon_{\Delta F, d}^{2}+\beta_{2}^{\Delta F} \sigma_{\Delta F, d \prime}^{2} \\
E_{d}\left[\varepsilon_{\Delta S, d+1} \varepsilon_{\Delta F, d+1}\right] \equiv \sigma_{\Delta S \Delta F, d+1}=\rho_{\Delta S \Delta F, d+1} \cdot \sigma_{\Delta S, d+1} \cdot \sigma_{\Delta F, d+1} \\
\rho_{\Delta S \Delta F, d+1}=\frac{q_{\Delta S \Delta F, d+1}}{\sqrt{q_{\Delta S \Delta S, d+1} \cdot q_{\Delta F \Delta F, d+1}}}, \\
q_{\Delta S \Delta F, d+1}=\bar{\rho}_{\Delta S \Delta F}+a_{1} \cdot\left(\varepsilon_{\Delta S, d} \cdot \varepsilon_{\Delta F, d}-\bar{\rho}_{\Delta S \Delta F}\right)+a_{2} \cdot\left(q_{\Delta S \Delta F, d}-\bar{\rho}_{\Delta S \Delta F}\right)
\end{gathered}
$$

In the above system of equations, $\Delta S_{d}$ and $\Delta F_{d}$ denote the daily spot and futures price changes over the past 1008 trading days up to day $d$, respectively, and $E_{d}[$.$] denotes the$ expectation operator conditional on day $d$ information. $\sigma_{\Delta S, d+1}^{2}$ is the day- $d$ expected condi- 
tional variance of daily spot price changes $(\Delta S), \sigma_{\Delta F, d+1}^{2}$ is the day- $d$ expected conditional variance of daily futures price changes $(\Delta F)$, and $\sigma_{\Delta S \Delta F, d+1}^{2}$ is the day-d expected conditional covariance between $\Delta S$ and $\Delta F \cdot \rho_{\Delta S \Delta F, d+1}=q_{\Delta S \Delta F, d+1} / \sqrt{q_{\Delta S \Delta S, d+1} \cdot q_{\Delta F \Delta F, d+1}}$ is the day- $d$ expected conditional correlation between $\Delta S$ and $\Delta F ; \bar{\rho}_{\Delta S \Delta F}$ is the unconditional correlation between $\Delta S$ and $\Delta F$. To ease the parameter convergence, we follow Bali and Engle (2010) and Bali et al. (2017) and use correlation targeting, assuming that the time-varying correlation mean reverts to the sample correlation, $\bar{\rho}_{\Delta S \Delta F}$. For each day $d+1$, the DCC hedge ratio is defined as the ratio of Equations (6) and (7): $h_{d c c}=\sigma_{\Delta S \Delta F, d+1} / \sigma_{\Delta F, d+1}^{2}$. Therefore, $h_{d c c}$ is purely out-of-sample, estimated using the information available up to the formation of the hedged portfolio (i.e., day $d$ ).

\subsection{The Simple Carry-Cost-Rate Hedge Ratio}

Unfortunately, the traditional hedge ratio ignores the economic connection between the spot and futures prices. Leistikow et al. (2020) derived a hedge ratio that incorporates the carry-cost hypothesis that links spot and futures prices as follows:

$$
S(1+c)^{T}=F
$$

where $c$ is the annualized spot asset's carry-cost rate (hereinafter CCR) to the futures' maturity, $T$ is the years to the futures contract's maturity, and $S$ and $F$ are the asset's contemporaneous spot and futures prices, respectively.

From the carry-cost hypothesis, it follows that $\frac{\partial S}{\partial F}=(1+c)^{-T}$; thus, $\frac{\sigma_{\Delta S}}{\sigma_{\Delta F}}=(1+c)^{-T}$. Assuming $\Delta S$ and $\Delta F$ are perfectly correlated, the day $d$ instantaneous hedge ratio $\left(h_{c c r}\right)$ is derived as $^{5}$ :

$$
h_{c c r}=(1+c)^{-T}
$$

Therefore, $h_{c c r}$ is calculated quickly and simply, since it only requires knowledge of $c$ and $T$ on day $d$.

\subsection{The Augmented Carry-Cost-Rate Hedge Ratios}

The simple CCR-based hedge ratio discussed in the previous subsection is easier to implement and has a firmer economic foundation than the statistics-based approaches discussed earlier. However, $h_{c c r}$ is biased upward because the correlation between $\Delta S$ and $\Delta F$ is almost certainly less than its assumed level of one. ${ }^{6}$ In this subsection, we introduce several ways to augment $h_{c c r}$ to correct its upward bias.

First, we augment $h_{c c r}$ with the dynamic conditional correlation between $\Delta S$ and $\Delta F$. It was estimated as part of the dynamic conditional hedge ratio discussed earlier in Section 2.2. We calculate this hedge ratio, denoted $h_{c c r \_d c c}$, on day $d$ as:

$$
h_{c c r \_d c c}=h_{c c r} \cdot \rho_{d c c},
$$

where $\rho_{d c c}$ comes from Equation (8) based on daily data over the period from $d$-1007 to day d.

Second, we augmented $h_{c c r}$ with the correlation $(\rho)$ between daily $\Delta S$ and $\Delta F$ over the period from $d-1007$ to day $d$. We calculated this hedge ratio, denoted $h_{c c r}$ corr, on day $d$ as:

$$
h_{c c r \_c o r r}=h_{c c r} \cdot \rho
$$

Finally, we use the Leistikow et al. (2020) BAM bias-adjusted $h_{c c r}$ on day $d$, denoted $h_{\text {ccr_bam: }}$ :

$$
h_{c c r \_b a m}=h_{c c r} \cdot B A M
$$

where $B A M$ is the bias-adjustment multiplier, defined as the average ratio of the average daily $h_{t}$ and average $h_{c c r}$ for each futures contract employed in the hedges over the 1008 trading days between day $d-1007$ to day $d$. In this way, each of the three bias-adjustment factors: 
$\rho_{d c c}, \rho$, and BAM, proxy $h_{c c r}$ 's bias based on the same amount of data, 1008 trading days for now.

\subsection{The "Naive" Hedge Ratio}

Despite the widespread acceptance of $h_{t}$ and the development of more complex statistical variants of it, Wang et al. (2015) find that $h_{t}$ and its more complex statistical variants do not reduce risk better than does the "naive" hedge ratio of $1\left(h_{1}\right)$. Therefore, we use $h_{1}$ as well as the regression-based hedge ratio $\left(h_{t}\right)$ as benchmarks.

\section{Data}

We study the S\&P500, Japanese Yen (JY), and gold since they represent a broad cross section of asset classes, their carry-cost rates are known and objectively calculable, and their spot and futures markets trade actively. All data are downloaded via a Bloomberg terminal, which are updated daily during our sample period.

CME e-mini S\&P500 futures contracts mature on the 3rd Friday of their maturity month and have been the most liquid equity futures since about 2000 when they overtook the regular S\&P500 futures. We study their March, June, September, and December contract maturities because they are the most liquid contract maturities and are very liquid (except when they are far from and very near to their maturities). These futures contract maturity months are alternatingly used as the hedging instrument where the hedging instrument is the near maturity futures contract until it is one week to its maturity at which point it switches to the second near maturity futures contract. The JY futures contracts mature two business days before the 3rd Wednesday in the maturity month. For liquidity reasons, the near futures maturity contract is used as the hedging instrument for the IMM Japanese Yen, until the 1st Friday of the maturity month. For COMEX gold, we use the liquid February, April, June, August, October, and December futures contract maturities and roll the hedging instrument from the near to the second near futures maturity two weeks earlier than for the JY due to gold's earlier liquidity shift from the near to the second near futures due to gold futures' delivery options. The gold futures mature the 3rd last business day of the maturity month.

The S\&P500 data begins with the Mar '98, while the JY data begins with the Mar '97, and the gold data begins with the Feb ' 91 futures contract as the hedging instrument. The Jun '20 futures is the last futures used as the hedging instrument for all the assets.

The carry-cost rate is the US risk-free short-term interest rate ${ }^{7}$ minus the S\&P500 dividend yield, the excess of the US over the JY risk-free short-term interest rate, and the US risk-free short-term interest rate, for the S\&P500, JY and gold, respectively.

\section{Statistics for the Hedge Ratios and Their Differences from the Benchmarks}

In this section, we provide statistics for the various daily profit hedge ratios (and their differences from the benchmark hedge ratios) discussed in Section 2. After the first 4 years (given the 4 years rolling window), for each subsequent S\&P500 and JY futures contract an average hedge ratio is calculated based on the 252/4 trading days that the contract is the hedging instrument since their contracts follow a quarterly rotation. The corresponding gold average hedge ratio is calculated based on the 252/6 trading days, since gold futures contracts follow a bimonthly rotation. These futures' average hedge ratios represent the underlying data for the Table 1 statistics. Table $1 \mathrm{~A}$ reports the descriptive statistics for the average hedge ratios for the S\&P500 in Panel A, JY in Panel B, and gold in Panel C. ${ }^{8}$ In each panel, the first column presents the results of the traditional regression hedge ratio $\left(h_{t}\right)$, which serves as Table 1A's benchmark hedge ratio. Columns 2-6 represent the descriptive statistics for the differences between $h_{t}$ and the: dynamic conditional hedge ratio $\left(h_{d c c}\right)$, the simple carry-cost-rate hedge ratio $\left(h_{c c r}\right)$, and, finally, the carry-cost-rate hedge ratio augmented with (i) the dynamic conditional correlation estimated from the DCC model $\left(h_{c c r} d c c\right)$, (ii) the simple correlation between the daily spot and futures price changes $\left(h_{c c r}\right.$ _corr $)$, and (iii) the bias-adjustment multiplier $\left(h_{c c r}\right.$ bam $)$, respectively. 
Column (1) of Table 1A shows that the mean traditional hedge ratios $\left(h_{t}\right)$ for the S\&P500, JY, and gold are between 0.8975 and 0.9568 . Their standard deviations are very small, ranging from 0.0151 to 0.0469 . Thus, as expected, their daily hedge ratios are highly persistent since they are estimated using 1008 trading-days rolling windows.

Table 1. The Benchmark Hedge Ratios and Differences from them.

\section{A. The Benchmark Hedge Ratio and Differences from it When $h_{t}$ is the Benchmark.}

\begin{tabular}{|c|c|c|c|c|c|c|}
\hline \multicolumn{7}{|c|}{ Panel A. S\&P500 } \\
\hline & $h_{t}$ & $h_{d c c}-h_{t}$ & $h_{c c r}-h_{t}$ & $h_{c c r \_d c c}-h_{t}$ & $h_{c c r \_c o r r}-h_{t}$ & $h_{c c r \_b a m}-h_{t}$ \\
\hline mean & 0.9568 & 0.0073 & 0.0441 & 0.0133 & 0.0153 & -0.0012 \\
\hline std & 0.0151 & 0.0323 & 0.0156 & 0.0164 & 0.014 & 0.0144 \\
\hline count & 74 & 74 & 74 & 74 & 74 & 58 \\
\hline t-stat & & 1.9382 & 24.3608 & 6.9605 & 9.3919 & -0.614 \\
\hline signif lev & & $10 \%$ & $1 \%$ & $1 \%$ & $1 \%$ & \\
\hline \multicolumn{7}{|c|}{ Panel B. JY } \\
\hline & $h_{t}$ & $h_{d c c}-h_{t}$ & $h_{c c r}-h_{t}$ & $h_{c c r-d c c}-h_{t}$ & $h_{c c r \_c o r r}-h_{t}$ & $h_{c c r \_b a m}-h_{t}$ \\
\hline mean & 0.9554 & -0.01 & 0.0423 & 0 & -0.0065 & -0.0036 \\
\hline std & 0.018 & 0.0389 & 0.0177 & 0.0232 & 0.0208 & 0.0162 \\
\hline count & 78 & 78 & 78 & 78 & 78 & 62 \\
\hline t-stat & & -2.2673 & 21.1487 & 0.0174 & -2.757 & -1.7318 \\
\hline signif lev & & $5 \%$ & $1 \%$ & & $1 \%$ & $10 \%$ \\
\hline \multicolumn{7}{|c|}{ Panel C. Gold } \\
\hline & $h_{t}$ & $h_{d c c}-h_{t}$ & $h_{c c r}-h_{t}$ & $h_{c c r_{-} d c c}-h_{t}$ & $h_{c c r \_c o r r}-h_{t}$ & $h_{c c r \_b a m}-h_{t}$ \\
\hline mean & 0.8975 & 0.0112 & 0.0982 & 0.0233 & 0.0098 & 0.0058 \\
\hline std & 0.0469 & 0.0582 & 0.0483 & 0.0387 & 0.0189 & 0.0287 \\
\hline count & 153 & 153 & 153 & 153 & 153 & 129 \\
\hline t-stat & & 2.3805 & 25.1193 & 7.4559 & 6.3829 & 2.3166 \\
\hline signif lev & & $5 \%$ & $1 \%$ & $1 \%$ & $1 \%$ & $5 \%$ \\
\hline \multicolumn{7}{|c|}{ Panel D. HR Differences Aggregated across Assets } \\
\hline & & $h_{d c c}-h_{t}$ & $h_{c c r}-h_{t}$ & $h_{c c r_{-} d c c}-h_{t}$ & $h_{c c r-c o r r}-h_{t}$ & $h_{c c r \_b a m}-h_{t}$ \\
\hline mean & & 0.0002 & 0.0715 & 0.0123 & 0.0084 & 0.0015 \\
\hline std & & 0.0429 & 0.0451 & 0.0238 & 0.0215 & 0.0156 \\
\hline count & & 222 & 222 & 222 & 222 & 174 \\
\hline t-stat & & 0.0775 & 23.619 & 7.6897 & 5.8467 & 1.2684 \\
\hline signif lev & & & $1 \%$ & $1 \%$ & $1 \%$ & \\
\hline
\end{tabular}

The count is the number of futures contracts used as hedge instruments, or, alternatively, the number of hedges since each hedge has a single hedging instrument. For each futures, the "average hedge ratio" is determined from the $\approx 252 / 4$ dynamic daily hedge ratios calculated for a quarterly maturing futures contract (e.g., as for the S\&P500 and JY, whereas for gold there are $\approx 252 / 6$ dynamic daily hedge ratios since the futures maturities are bimonthly). Since the BAM is calculated from 4 years of futures contracts, the $h_{\text {ccr_bam }}$ count is $4 \times 4(6 \times 4)$ for the quarterly futures contract maturities of the S\&P500 and JY (bimonthly maturities of gold).

The "aggregated across assets" count is equal-weighted. It uses the most recent differences for each asset for roughly the same period as the e-mini S\&P500 (since its data series is the shortest). Since the gold futures data started earlier and there are $50 \%$ more gold futures maturities/years, a little less than the 1st half of the gold futures contract results do not enter into the aggregation across assets. 
Table 1. Cont.

B. The Benchmark Hedge Ratio and Differences from it When $h_{1}$ is the Benchmark

Panel A. S\&P500

\begin{tabular}{|c|c|c|c|c|c|c|}
\hline & $h_{1}$ & $h_{d c c}-h_{1}$ & $h_{c c r}-h_{1}$ & $h_{c c r_{-} d c c}-h_{1}$ & $h_{c c r \_c o r r}-h_{1}$ & $h_{c c r \_b a m}-h_{1}$ \\
\hline mean & 1 & -0.0359 & 0.0009 & -0.0299 & -0.0279 & -0.0396 \\
\hline std & 0 & 0.0321 & 0.0024 & 0.0058 & 0.0054 & 0.0106 \\
\hline count & 74 & 74 & 74 & 74 & 74 & 58 \\
\hline t-stat & & -9.6302 & 3.3772 & -44.7285 & -44.1444 & -28.4181 \\
\hline signif lev & & $1 \%$ & $1 \%$ & $1 \%$ & $1 \%$ & $1 \%$ \\
\hline \multicolumn{7}{|c|}{ Panel B. JY } \\
\hline & $h_{1}$ & $h_{d c c}-h_{1}$ & $h_{c c r}-h_{1}$ & $h_{c c r_{-} d c c}-h_{1}$ & $h_{c c r \_c o r r}-h_{1}$ & $h_{c c r \_b a m}-h_{1}$ \\
\hline mean & 1 & -0.0546 & -0.0023 & -0.0446 & -0.0511 & -0.0431 \\
\hline std & 0 & 0.0391 & 0.0025 & 0.0156 & 0.0132 & 0.0123 \\
\hline count & 78 & 78 & 78 & 78 & 78 & 62 \\
\hline t-stat & & -12.3317 & -8.2224 & -25.2679 & -34.2633 & -27.5771 \\
\hline signif lev & & $1 \%$ & $1 \%$ & $1 \%$ & $1 \%$ & $1 \%$ \\
\hline \multicolumn{7}{|c|}{ Panel C. Gold } \\
\hline & $h_{1}$ & $h_{d c c}-h_{1}$ & $h_{c c r}-h_{1}$ & $h_{c c r-d c c}-h_{1}$ & $h_{c c r \_c o r r}-h_{1}$ & $h_{c c r \_b a m}-h_{1}$ \\
\hline mean & 1 & -0.0913 & -0.0043 & -0.0791 & -0.0927 & -0.0913 \\
\hline std & 0 & 0.0671 & 0.0039 & 0.0385 & 0.0465 & 0.0362 \\
\hline count & 153 & 153 & 153 & 153 & 153 & 129 \\
\hline t-stat & & -16.8137 & -13.8224 & -25.4034 & -24.6593 & -28.6446 \\
\hline signif lev & & $1 \%$ & $1 \%$ & $1 \%$ & $1 \%$ & $1 \%$ \\
\hline \multicolumn{7}{|c|}{ Panel D. HR Differences Aggregated across Assets } \\
\hline & & $h_{d c c}-h_{1}$ & $h_{c c r}-h_{1}$ & $h_{c c r-d c c}-h_{1}$ & $h_{c c r \_c o r r}-h_{1}$ & $h_{c c r \_b a m}-h_{1}$ \\
\hline mean & & -0.0721 & -0.0008 & -0.06 & -0.0639 & -0.0694 \\
\hline std & & 0.059 & 0.0024 & 0.0368 & 0.039 & 0.0422 \\
\hline count & & 222 & 222 & 222 & 222 & 174 \\
\hline t-stat & & -18.2127 & -4.9113 & -24.2818 & -24.4085 & -21.6855 \\
\hline signif lev & & $1 \%$ & $1 \%$ & $1 \%$ & $1 \%$ & $1 \%$ \\
\hline
\end{tabular}

The count is the number of futures contracts used as hedge instruments, or, alternatively, the number of hedges since each hedge has a single hedging instrument. For each futures, the "average hedge ratio" is determined from the $\approx 252 / 4$ dynamic daily hedge ratios calculated for a quarterly maturing futures contract (e.g., as for the S\&P500 and JY, whereas for gold there are $\approx 252 / 6$ dynamic daily hedge ratios since the futures maturities are bimonthly). Since the BAM is calculated from 4 years of futures contracts, the $\mathrm{h}_{\text {ccr_bam }}$ count is $4 \times 4(6 \times 4)$ for the quarterly futures contract maturities of the S\&P500 and JY (bimonthly maturities of gold).

The "aggregated across assets" count is equal-weighted. It uses the most recent differences for each asset for roughly the same period as the e-mini S\&P500 (since its data series is the shortest). Since the gold futures data started earlier and there are $50 \%$ more gold futures maturities/years, a little less than the 1st half of the gold futures contract results do not enter into the aggregation across assets.

Columns 2-6 of Table 1A show that the mean differences between the other hedge ratios and $h_{t}$ are small $(<0.0233$ in absolute value across the assets); except for the upwardbiased $h_{c c r}$; its maximum mean difference is 0.0982 (for gold). Therefore, the bias adjustments to the CCR hedge ratio largely mitigate the simple CCR hedge ratio's upward bias. Similar to $h_{c c r}$, both $\mathrm{h}_{\mathrm{ccr} \_\mathrm{dcc}}$ and $h_{\mathrm{ccr} \_ \text {corr }}$ are larger than $h_{t}$ and the difference is significant at the $1 \%$ level; however, this does not hold for the JY. The paper's statistical significance 
tests are two-tailed tests, except for the one-tailed $h_{c c r}-h_{t}$ test in that $h_{c c r}$ is upward biased relative to $h_{t}$.

Table 1A, Panel D has equal-weighted, roughly contemporaneous, aggregated across asset results that end with the assets' June 2020 futures contracts. To generate these results, since the S\&P500 data/results start later than the other assets' (starting with the Mar 2002 futures), all of its results are included but only the contemporaneous JY (starting with the Mar 2002 futures) and roughly contemporaneous gold results are included in the aggregation (starting with the April 2008 futures). The gold results included in the equal-weighted aggregation begin later, since gold has 50\% more futures maturities/years than the others and therefore gets the same number of futures contracts in $2 / 3$ the time. The aggregated across assets mean $h_{c c r}-h_{t}$ was above 0.07 , while the means for the other differences were not more than 0.0123 in absolute value. Hedge ratios $h_{c c r}, h_{c c r} d c c$, and $h_{c c r}$ corr are all larger than $h_{t}$ at the $1 \%$ significance level.

Table $1 \mathrm{~B}$ below is the same as Table $1 \mathrm{~A}$, except that Table 1B's benchmark hedge ratio is $h_{1}$. The other hedge ratios' means, except for the S\&P500 $h_{c c r}$, are significantly less than 1 at the $1 \%$ confidence level for all assets. For the Panel D, aggregations across assets, while $h_{c c r}$ 's mean is barely below 1 , the other hedge ratios' means are between 0.06 and 0.0721 below 1 . All of the hedge ratio means are below $h_{1}$ at the $1 \%$ significance level. The fact that, for this study period and these assets, the upward biased $h_{c c r}$ is significantly below 1 portends poor $h_{1}$ hedging performance; this conjecture is strongly supported by the results in the next section.

\section{The Benchmark Hedge Ratios' Hedging Performances and Hedging Performance Differences from Those of the Benchmark Hedge Ratios}

Next, we calculated each hedge ratio's out-of-sample hedge effectiveness $(H E)$ and compared it to those of the two benchmark hedge ratios. The $H E$ is defined as the percentage profit variance reduction for each contract over our sample period:

$$
\begin{gathered}
H E=1-\frac{\operatorname{Var}(\text { hedged profits })}{\operatorname{Var}(\text { unhedged profits })} \\
\text { Hedged profit }=\Delta S-h \cdot \Delta F \\
\text { Unhedged profit }=\Delta S
\end{gathered}
$$

where $\operatorname{Var}($.$) denotes variance, \Delta S$ and $\Delta F$ are, respectively, the daily spot and futures price changes on day $d+1$, and $h$ denotes a hedge ratio on day $d$ calculated from one of the hedge ratios described in Section 2. Therefore, the HE of a hedge ratio increases with the risk reduced.

Table 2A reports the hedge effectiveness results, where $h_{t}$ 's HE is the benchmark. Column 1 is $h_{t}$ 's HE while the remaining columns represent the hedge effectiveness differences in between those for the various hedge ratios and for $h_{t}$.

Table 2A, column (1) shows that $h_{t}$ 's average HE for the S\&P500, JY, and gold are between 0.8464 and 0.9388 . Their corresponding standard deviations are small, ranging from 0.0378 to 0.1477 . These results indicate that $h_{t}$ 's out-of-sample HE is highly persistent.

The HE for $h_{t}$ is higher than that for either $h_{d c c}$ or $h_{c c r}$. The latter result suggests that the benefit of $h_{c c r}$ through recognizing the economic link between the spot and the future prices is less than the cost of its upward bias. The finding that $h_{d c c}$ does not outperform $h_{t}$ is consistent with previous studies that show that complicated time-series hedge ratios do not yield superior HE performance. ${ }^{9}$ Therefore, modeling the dynamic conditional hedge ratio based on spot and futures price changes alone is insufficient to improve hedge effectiveness.

Columns 4-6 of Table 2A compare the augmented $h_{c c r} s^{\prime}$ HEs with $h_{t^{\prime} \mathrm{s}}$ HE where the $h_{c c r}$ is augmented with the conditional correlation $\left(h_{c c r \_} d c c\right)$, the unconditional correlation $\left(h_{c c r \_c o r r}\right)$, and the bias-adjustment multiplier $\left(h_{c c r}\right.$ bam $)$, respectively. Column (4) shows that the HE for $h_{c c r_{-} d c c}$ is higher than that for $h_{t}$ for each asset, where the difference in HE is generally statistically significant at the 5\% level. When the HE differences are aggregated across assets, 
the HE for $h_{c c r} \_d c c$ is statistically significantly higher than that for $h_{t}$ at the $1 \%$ significant level. Columns (5) and (6) show that the HEs for $h_{\text {ccr_corr }}$ and $h_{\text {ccr_bam }}$ are not typically significantly different from that for $h_{t}$. Therefore, the $h_{c c r \_d c c}$ result suggests that incorporating the economic relation between the spot and futures prices and properly modeling their time-varying conditional correlations jointly produce the best hedging performance.

Table 2. The Benchmark HRs' Hedge Effectiveness and Differences from them.

\begin{tabular}{|c|c|c|c|c|c|c|}
\hline \multicolumn{7}{|c|}{ A. The Benchmark HR's Hedge Effectiveness and Differences from it When $h_{t}$ is the Benchmark } \\
\hline \multicolumn{7}{|c|}{ Panel A. S\&P500 } \\
\hline & $h_{t}$ & $h_{d c c}-h_{t}$ & $h_{c c r}-h_{t}$ & $h_{c c r-d c c}-h_{t}$ & $h_{c c r-c o r r}-h_{t}$ & $h_{c c r \_b a m}-h_{t}$ \\
\hline mean & 0.9388 & -0.0014 & -0.0011 & 0.0035 & 0.0003 & 0.0001 \\
\hline std & 0.0378 & 0.0215 & 0.005 & 0.0137 & 0.0022 & 0.002 \\
\hline count & 74 & 74 & 74 & 74 & 74 & 58 \\
\hline t-stat & & -0.5447 & -1.9024 & 2.1813 & 1.1068 & 0.2169 \\
\hline signif lev & & & $10 \%$ & $5 \%$ & & \\
\hline \multicolumn{7}{|c|}{ Panel B. JY } \\
\hline & $h_{t}$ & $h_{d c c}-h_{t}$ & $h_{c c r}-h_{t}$ & $h_{c c r-d c c}-h_{t}$ & $h_{c c r-c o r r}-h_{t}$ & $h_{c c r \_b a m}-h_{t}$ \\
\hline mean & 0.9107 & -0.0023 & -0.0018 & 0.0003 & 0.0008 & 0.0001 \\
\hline std & 0.0479 & 0.0098 & 0.0051 & 0.0028 & 0.003 & 0.0022 \\
\hline count & 78 & 78 & 78 & 78 & 78 & 62 \\
\hline t-stat & & -2.0244 & -3.1242 & 1.0111 & 2.2843 & 0.5062 \\
\hline signif lev & & $5 \%$ & $1 \%$ & & $5 \%$ & \\
\hline \multicolumn{7}{|c|}{ Panel C. Gold } \\
\hline & $h_{t}$ & $h_{d c c}-h_{t}$ & $h_{c c r}-h_{t}$ & $h_{c c r_{-} d c c}-h_{t}$ & $h_{c c r \_c o r r}-h_{t}$ & $h_{c c r-b a m}-h_{t}$ \\
\hline mean & 0.8464 & -0.0184 & -0.0115 & 0.0055 & -0.0006 & -0.0006 \\
\hline std & 0.1477 & 0.1955 & 0.026 & 0.0259 & 0.0046 & 0.0071 \\
\hline count & 153 & 153 & 153 & 153 & 153 & 129 \\
\hline t-stat & & -1.1639 & -5.4726 & 2.6309 & -1.5674 & -0.9196 \\
\hline signif lev & & & $1 \%$ & $1 \%$ & & \\
\hline \multicolumn{7}{|c|}{ Panel D. HE Differences Aggregated across Assets } \\
\hline & & $h_{d c c}-h_{t}$ & $h_{c c r}-h_{t}$ & $h_{c c r-d c c}-h_{t}$ & $h_{c c r-c o r r}-h_{t}$ & $h_{c c r-b a m}-h_{t}$ \\
\hline mean & & -0.0023 & -0.0085 & 0.0032 & 0 & 0.0001 \\
\hline std & & 0.0189 & 0.0197 & 0.0124 & 0.0041 & 0.0022 \\
\hline count & & 222 & 222 & 222 & 222 & 174 \\
\hline t-stat & & -1.8015 & -6.4151 & 3.8774 & -0.1053 & 0.4399 \\
\hline signif lev & & $10 \%$ & $1 \%$ & $1 \%$ & & \\
\hline \multicolumn{7}{|c|}{ B. The Benchmark HR's Hedge Effectiveness and Differences from it When $h_{1}$ is the Benchmark } \\
\hline \multicolumn{7}{|c|}{ Panel A. S\&P500 } \\
\hline & $h_{1}$ & $h_{d c c}-h_{1}$ & $h_{c c r}-h_{1}$ & $h_{c c r-d c c}-h_{1}$ & $h_{c c r \_c o r r}-h_{1}$ & $h_{c c r \_b a m}-h_{1}$ \\
\hline mean & 0.9378 & -0.0004 & -0.0002 & 0.0044 & 0.0012 & 0.0015 \\
\hline std & 0.0406 & 0.0228 & 0.0005 & 0.0167 & 0.003 & 0.0047 \\
\hline count & 74 & 74 & 74 & 74 & 74 & 58 \\
\hline t-stat & & -0.1566 & -2.6257 & 2.2837 & 3.5766 & 2.3963 \\
\hline signif lev & & & $5 \%$ & $5 \%$ & $1 \%$ & $5 \%$ \\
\hline
\end{tabular}


Table 2. Cont.

\begin{tabular}{|c|c|c|c|c|c|c|}
\hline \multicolumn{7}{|c|}{ Panel B. JY } \\
\hline & $h_{1}$ & $h_{d c c}-h_{1}$ & $h_{c c r}-h_{1}$ & $h_{c c r-d c c}-h_{1}$ & $h_{c c r \_c o r r}-h_{1}$ & $h_{c c r \_b a m}-h_{1}$ \\
\hline mean & 0.9087 & -0.0003 & 0.0002 & 0.0023 & 0.0028 & 0.0019 \\
\hline std & 0.0492 & 0.0126 & 0.0004 & 0.0057 & 0.0071 & 0.0055 \\
\hline count & 78 & 78 & 78 & 78 & 78 & 62 \\
\hline t-stat & & -0.1944 & 4.2624 & 3.554 & 3.4394 & 2.7357 \\
\hline signif lev & & & $1 \%$ & $1 \%$ & $1 \%$ & $1 \%$ \\
\hline \multicolumn{7}{|c|}{ Panel C. Gold } \\
\hline & $h_{1}$ & $h_{d c c}-h_{1}$ & $h_{c c r}-h_{1}$ & $h_{c c r-d c c}-h_{1}$ & $h_{c c r-c o r r}-h_{1}$ & $h_{c c r-b a m}-h_{1}$ \\
\hline mean & 0.8343 & -0.0063 & 0.0006 & 0.0176 & 0.0115 & 0.014 \\
\hline std & 0.1629 & 0.1958 & 0.0011 & 0.0373 & 0.0251 & 0.0219 \\
\hline count & 153 & 153 & 153 & 153 & 153 & 129 \\
\hline t-stat & & -0.3963 & 7.3019 & 5.8403 & 5.692 & 7.2647 \\
\hline signif lev & & & $1 \%$ & $1 \%$ & $1 \%$ & $1 \%$ \\
\hline \multicolumn{7}{|c|}{ Panel D. HE Differences Aggregated across Assets } \\
\hline & & $h_{d c c}-h_{1}$ & $h_{c c r}-h_{1}$ & $h_{c c r_{-} d c c}-h_{1}$ & $h_{c c r \_c o r r}-h_{1}$ & $h_{c c r \_b a m}-h_{1}$ \\
\hline mean & & 0.0063 & 0.0001 & 0.0118 & 0.0086 & 0.0096 \\
\hline std & & 0.0296 & 0.0005 & 0.0249 & 0.0184 & 0.0185 \\
\hline count & & 222 & 222 & 222 & 222 & 174 \\
\hline t-stat & & 3.1852 & 4.0726 & 7.0864 & 6.9614 & 6.8561 \\
\hline signif lev & & $1 \%$ & $1 \%$ & $1 \%$ & $1 \%$ & $1 \%$ \\
\hline
\end{tabular}

Table 2B reports the same results; however, $h_{1}$ 's HE is the benchmark. The HE for $h_{1}$ is statistically significantly lower (generally at the $1 \%$ level) than that for all the other hedge ratios except for $h_{d c c}$ and, for the S\&P500, $h_{c c r}$. Our finding differs from that of Wang et al. (2015) and neither should be interpreted as a general result. The low HE for $h_{1}$ was anticipated earlier when we noted that the upward biased $h_{c c r}$ was significantly less than 1. Our low HE for $h_{1}$ is not a general result since the carry-cost rate varies across assets, currency denominations, and time; thus, in the very improbable case that the upward biased $h_{c c r}$ exceeded 1 by its bias, the risk minimizing hedge ratio would be $h_{1}$.

Table 3 repeats the HE analyses, except that it analyzes the first and second halves of our sample period separately to see if the HE and HE difference results are stable across subperiods. The only HE difference from the benchmark's HE that is nearly always statistically significantly positive across both benchmarks, both halves, and all 3 assets is that for $h_{c c r} d c c$ and the JY in the 1st half when $h_{t}$ is the benchmark is the main exception. The HEs for the other two augmented $h_{c c r} \mathrm{~s}$ are statistically significantly higher than those for $h_{1}$ for 8 of the 12 individual asset results. However, their HEs are not generally higher for those of $h_{t}$. Table 3, Panel D, shows for the aggregations across assets that the HE for $h_{c c r} d c c$ is statistically significantly higher than that for either benchmark in both sub-periods at the $1 \%$ level. The HEs for the other two augmented $h_{c c r}$ s are also statistically significantly higher than those for $h_{1}$ in both halves at the $1 \%$ level. 
Table 3. Stability of the Benchmark HRs' HEs and Differences from them.

\section{Panel A. S\&P500}

1st half: HE and HE differences when the benchmark is $h_{t}$

\begin{tabular}{|c|c|c|c|c|c|c|}
\hline & $h_{t}$ & $h_{d c c}-h_{t}$ & $h_{c c r}-h_{t}$ & $h_{c c r \_d c c}-h_{t}$ & $h_{c c r \_c o r r}-h_{t}$ & $h_{c c r \_b a m}-h_{t}$ \\
\hline mean & 0.9427 & -0.0007 & 0.0003 & 0.0013 & 0.0008 & 0.0001 \\
\hline std & 0.0248 & 0.0045 & 0.0036 & 0.0028 & 0.0017 & 0.0022 \\
\hline count & 37 & 37 & 37 & 37 & 37 & 21 \\
\hline t-stat & & -0.9852 & 0.493 & 2.8216 & 3.0666 & 0.2467 \\
\hline signif lev & & & & $1 \%$ & $1 \%$ & \\
\hline
\end{tabular}

2nd half: HE and HE differences when the benchmark is $h_{t}$

\begin{tabular}{|c|c|c|c|c|c|c|}
\hline & $h_{t}$ & $h_{d c c}-h_{t}$ & $h_{c c r}-h_{t}$ & $h_{c c r-d c c}-h_{t}$ & $h_{c c r \_c o r r}-h_{t}$ & $h_{c c r \_b a m}-h_{t}$ \\
\hline mean & 0.9348 & -0.002 & -0.0025 & 0.0057 & -0.0003 & 0 \\
\hline std & 0.0475 & 0.0303 & 0.0058 & 0.019 & 0.0026 & 0.0019 \\
\hline count & 37 & 37 & 37 & 37 & 37 & 37 \\
\hline t-stat & & -0.3998 & -2.6145 & 1.8057 & -0.6361 & 0.0712 \\
\hline signif lev & & & $5 \%$ & $10 \%$ & & \\
\hline \multicolumn{7}{|c|}{ 1st half: HE and HE differences when the benchmark is $h_{1}$} \\
\hline & $h_{1}$ & $h_{d c c}-h_{1}$ & $h_{c c r}-h_{1}$ & $h_{c c r \_d c c}-h_{1}$ & $h_{c c r \_c o r r}-h_{1}$ & $h_{c c r \_b a m}-h_{1}$ \\
\hline mean & 0.9429 & -0.001 & 0.0001 & 0.0011 & 0.0006 & 0.0003 \\
\hline std & 0.0256 & 0.0042 & 0.0003 & 0.0039 & 0.0026 & 0.0037 \\
\hline count & 37 & 37 & 37 & 37 & 37 & 21 \\
\hline t-stat & & -1.4123 & 1.0029 & 1.6317 & 1.4325 & 0.3594 \\
\hline
\end{tabular}

signif lev

2nd half: HE and HE differences when the benchmark is $h_{1}$

\begin{tabular}{|c|c|c|c|c|c|c|}
\hline & $h_{1}$ & $h_{d c c}-h_{1}$ & $h_{c c r}-h_{1}$ & $h_{c c r \_d c c}-h_{1}$ & $h_{c c r \_c o r r}-h_{1}$ & $h_{c c r-b a m}-h_{1}$ \\
\hline mean & 0.9327 & 0.0001 & -0.0004 & 0.0078 & 0.0019 & 0.0022 \\
\hline std & 0.0513 & 0.0322 & 0.0005 & 0.0229 & 0.0032 & 0.0051 \\
\hline count & 37 & 37 & 37 & 37 & 37 & 37 \\
\hline t-stat & & 0.0259 & -3.9637 & 2.0694 & 3.5117 & 2.5698 \\
\hline signif lev & & & $1 \%$ & $5 \%$ & $1 \%$ & $5 \%$ \\
\hline
\end{tabular}

Panel B. JY

1st half: HE and HE differences when the benchmark is $h_{t}$

\begin{tabular}{|c|c|c|c|c|c|c|}
\hline & $h_{t}$ & $h_{d c c}-h_{t}$ & $h_{c c r}-h_{t}$ & $h_{c c r \_d c c}-h_{t}$ & $h_{c c r \_c o r r}-h_{t}$ & $h_{c c r \_b a m}-h_{t}$ \\
\hline mean & 0.9072 & -0.0019 & -0.002 & 0 & 0.0008 & -0.0002 \\
\hline std & 0.0553 & 0.0117 & 0.0059 & 0.0034 & 0.0036 & 0.0013 \\
\hline count & 39 & 39 & 39 & 39 & 39 & 23 \\
\hline t-stat & & -0.9886 & -2.1761 & -0.065 & 1.3773 & -0.8266 \\
\hline signif lev & & & $5 \%$ & & & \\
\hline
\end{tabular}


Table 3. Cont.

\begin{tabular}{|c|c|c|c|c|c|c|}
\hline \multicolumn{7}{|c|}{ 2nd half: HE and HE differences when the benchmark is $h_{t}$} \\
\hline & $h_{t}$ & $h_{d c c}-h_{t}$ & $h_{c c r}-h_{t}$ & $h_{c c r-d c c}-h_{t}$ & $h_{c c r-c o r r}-h_{t}$ & $h_{c c r-b a m}-h_{t}$ \\
\hline mean & 0.9142 & -0.0026 & -0.0015 & 0.0007 & 0.0008 & 0.0004 \\
\hline std & 0.0397 & 0.0076 & 0.0041 & 0.002 & 0.0023 & 0.0026 \\
\hline count & 39 & 39 & 39 & 39 & 39 & 39 \\
\hline t-stat & & -2.1798 & -2.3064 & 2.0882 & 2.0626 & 0.8715 \\
\hline signif lev & & $5 \%$ & $5 \%$ & $5 \%$ & $5 \%$ & \\
\hline \multicolumn{7}{|c|}{ 1st half: HE and HE differences when the benchmark is $h_{1}$} \\
\hline & $h_{1}$ & $h_{d c c}-h_{1}$ & $h_{c c r}-h_{1}$ & $h_{c c r-d c c}-h_{1}$ & $h_{c c r-c o r r}-h_{1}$ & $h_{c c r-b a m}-h_{1}$ \\
\hline mean & 0.9049 & 0.0004 & 0.0002 & 0.0022 & 0.0031 & 0.0017 \\
\hline std & 0.0574 & 0.0148 & 0.0005 & 0.0067 & 0.0085 & 0.0066 \\
\hline count & 39 & 39 & 39 & 39 & 39 & 23 \\
\hline t-stat & & 0.1703 & 2.804 & 2.0646 & 2.2411 & 1.248 \\
\hline signif lev & & & $1 \%$ & $5 \%$ & $5 \%$ & \\
\hline \multicolumn{7}{|c|}{ 2nd half: HE and HE differences when the benchmark is $h_{1}$} \\
\hline & $h_{1}$ & $h_{d c c}-h_{1}$ & $h_{c c r}-h_{1}$ & $h_{c c r-d c c}-h_{1}$ & $h_{c c r \_c o r r}-h_{1}$ & $h_{c c r \_b a m}-h_{1}$ \\
\hline mean & 0.9126 & -0.001 & 0.0002 & 0.0024 & 0.0024 & 0.002 \\
\hline std & 0.0398 & 0.01 & 0.0003 & 0.0046 & 0.0053 & 0.0049 \\
\hline count & 39 & 39 & 39 & 39 & 39 & 39 \\
\hline t-stat & & -0.601 & 3.7447 & 3.2522 & 2.8669 & 2.6087 \\
\hline signif lev & & & $1 \%$ & $1 \%$ & $1 \%$ & $5 \%$ \\
\hline \multicolumn{7}{|c|}{ Panel C. Gold } \\
\hline \multicolumn{7}{|c|}{ 1st half: HE and HE differences when the benchmark is $h_{t}$} \\
\hline & $h_{t}$ & $h_{d c c}-h_{t}$ & $h_{c c r}-h_{t}$ & $h_{c c r-d c c}-h_{t}$ & $h_{c c r \_c o r r}-h_{t}$ & $h_{c c r \_b a m}-h_{t}$ \\
\hline mean & 0.9013 & -0.0333 & -0.0012 & 0.0053 & -0.0001 & -0.0007 \\
\hline std & 0.155 & 0.2766 & 0.018 & 0.0334 & 0.0029 & 0.0051 \\
\hline count & 76 & 76 & 76 & 76 & 76 & 52 \\
\hline t-stat & & -1.051 & -0.5814 & 1.3761 & -0.2459 & -0.9525 \\
\hline signif lev & & & & & & \\
\hline \multicolumn{7}{|c|}{ 2nd half: HE and HE differences when the benchmark is $h_{t}$} \\
\hline & $h_{t}$ & $h_{d c c}-h_{t}$ & $h_{c c r}-h_{t}$ & $h_{c c r-d c c}-h_{t}$ & $h_{c c r-c o r r}-h_{t}$ & $h_{c c r \_b a m}-h_{t}$ \\
\hline mean & 0.7922 & -0.0036 & -0.0216 & 0.0058 & -0.0011 & -0.0005 \\
\hline std & 0.118 & 0.0226 & 0.0286 & 0.0156 & 0.0057 & 0.0082 \\
\hline count & 77 & 77 & 77 & 77 & 77 & 77 \\
\hline t-stat & & -1.4157 & -6.6423 & 3.2315 & -1.6364 & -0.5428 \\
\hline signif lev & & & $1 \%$ & $1 \%$ & & \\
\hline \multicolumn{7}{|c|}{ 1st half: HE and HE differences when the benchmark is $h_{1}$} \\
\hline & $h_{1}$ & $h_{d c c}-h_{1}$ & $h_{c c r}-h_{1}$ & $h_{c c r-d c c}-h_{1}$ & $h_{c c r-c o r r}-h_{1}$ & $h_{c c r \_b a m}-h_{1}$ \\
\hline mean & 0.8993 & -0.0313 & 0.0009 & 0.0073 & 0.002 & 0.0028 \\
\hline std & 0.1648 & 0.2734 & 0.0014 & 0.0385 & 0.0198 & 0.0108 \\
\hline
\end{tabular}


Table 3. Cont.

\begin{tabular}{|c|c|c|c|c|c|c|}
\hline \multicolumn{7}{|c|}{ 1st half: HE and HE differences when the benchmark is $h_{1}$} \\
\hline count & 76 & 76 & 76 & 76 & 76 & 52 \\
\hline t-stat & & -0.9975 & 5.4563 & 1.6599 & 0.8745 & 1.8718 \\
\hline signif lev & \multicolumn{4}{|c|}{$1 \%$} & & $10 \%$ \\
\hline \multicolumn{7}{|c|}{ 2nd half: HE and HE differences when the benchmark is $h_{1}$} \\
\hline & $h_{1}$ & $h_{d c c}-h_{1}$ & $h_{c c r}-h_{1}$ & $h_{c c r-d c c}-h_{1}$ & $h_{c c r \_c o r r}-h_{1}$ & $h_{c c r \_b a m}-h_{1}$ \\
\hline mean & 0.7701 & 0.0184 & 0.0004 & 0.0278 & 0.021 & 0.0215 \\
\hline std & 0.1337 & 0.0411 & 0.0006 & 0.0334 & 0.0263 & 0.0242 \\
\hline count & 77 & 77 & 77 & 77 & 77 & 77 \\
\hline t-stat & & 3.9315 & 6.0318 & 7.3037 & 6.9998 & 7.8227 \\
\hline signif lev & & $1 \%$ & $1 \%$ & $1 \%$ & $1 \%$ & $1 \%$ \\
\hline \multicolumn{7}{|c|}{ Panel D. HE Differences Aggregated across Assets: } \\
\hline \multicolumn{7}{|c|}{ 1st half: HE differences when the benchmark is $h_{t}$} \\
\hline & $h_{d c c}-h_{t}$ & $h_{c c r}-h_{t}$ & $h_{c c r \_d c c}-h_{t}$ & $h_{c c r \_c o r r}-h_{t}$ & \multicolumn{2}{|c|}{$h_{c c r \_b a m}-h_{t}$} \\
\hline Mean & -0.0045 & -0.0066 & 0.0011 & 0.0005 & \multicolumn{2}{|c|}{0.0002} \\
\hline std & 0.016 & 0.0189 & 0.0062 & 0.0036 & \multicolumn{2}{|c|}{0.002} \\
\hline count & 111 & 111 & 111 & 111 & \multicolumn{2}{|c|}{63} \\
\hline t-stat & -2.9776 & -3.7015 & 1.9475 & 1.3209 & \multicolumn{2}{|c|}{0.7654} \\
\hline signif lev & $1 \%$ & $1 \%$ & $10 \%$ & & & \\
\hline \multicolumn{7}{|c|}{ 2nd half: HE differences when the benchmark is $h_{t}$} \\
\hline & $h_{d c c}-h_{t}$ & $h_{c c r}-h_{t}$ & $h_{c c r-d c c}-h_{t}$ & $h_{c c r \_c o r r}-h_{t}$ & \multicolumn{2}{|c|}{$h_{c c r \_b a m}-h_{t}$} \\
\hline mean & -0.0001 & -0.0103 & 0.0053 & -0.0005 & \multicolumn{2}{|c|}{0} \\
\hline std & 0.0213 & 0.0204 & 0.0162 & 0.0044 & \multicolumn{2}{|c|}{0.0024} \\
\hline count & 111 & 111 & 111 & 111 & \multicolumn{2}{|c|}{111} \\
\hline t-stat & -0.028 & -5.3351 & 3.4564 & -1.2058 & \multicolumn{2}{|c|}{0.0331} \\
\hline signif lev & & $1 \%$ & $1 \%$ & & & \\
\hline \multicolumn{7}{|c|}{ 1st half: HE differences when the benchmark is $h_{1}$} \\
\hline & $h_{d c c}-h_{1}$ & $h_{c c r}-h_{1}$ & $h_{c c r \_d c c}-h_{1}$ & $h_{c c r \_c o r r}-h_{1}$ & \multicolumn{2}{|c|}{$h_{c c r \_b a m}-h_{1}$} \\
\hline mean & 0.0023 & 0.0001 & 0.0079 & 0.0072 & \multicolumn{2}{|c|}{0.0082} \\
\hline std & 0.0255 & 0.0003 & 0.0198 & 0.0193 & \multicolumn{2}{|c|}{0.0165} \\
\hline count & 111 & 111 & 111 & 111 & \multicolumn{2}{|c|}{63} \\
\hline t-stat & 0.931 & 4.322 & 4.2188 & 3.9366 & \multicolumn{2}{|c|}{3.9247} \\
\hline signif lev & & $1 \%$ & $1 \%$ & $1 \%$ & \multicolumn{2}{|c|}{$1 \%$} \\
\hline \multicolumn{7}{|c|}{ 2nd half: HE differences when the benchmark is $h_{1}$} \\
\hline & $h_{d c c}-h_{1}$ & $h_{c c r}-h_{1}$ & $h_{c c r \_d c c}-h_{1}$ & $h_{c c r \_c o r r}-h_{1}$ & \multicolumn{2}{|c|}{$h_{c c r \_b a m}-h_{1}$} \\
\hline mean & 0.0104 & 0.0001 & 0.0158 & 0.01 & \multicolumn{2}{|c|}{0.0105} \\
\hline std & 0.0329 & 0.0006 & 0.0287 & 0.0174 & & 96 \\
\hline count & 111 & 111 & 111 & 111 & & \\
\hline t-stat & 3.3385 & 2.4052 & 5.7886 & 6.0397 & & 34 \\
\hline signif lev & $1 \%$ & $5 \%$ & $1 \%$ & $1 \%$ & & \\
\hline
\end{tabular}


Table 4 directly tests the HE differences across the various bias-adjusted $h_{c c r}$ approaches. The HE is statistically significantly higher for $h_{c c r} d_{c c}$ than it is for the other bias-adjustment approaches for the individual assets (other than for the JY) and for the aggregation across assets.

Table 4. Hedge Effectiveness Differences across $h_{c c r}$ Bias-Adjustment Methods.

\begin{tabular}{|c|c|c|c|}
\hline \multicolumn{4}{|c|}{ Panel A. S\&P500 } \\
\hline & $h_{c c r-d c c}-h_{c c r \_c o r r}$ & $h_{c c r-d c c}-h_{c c r \_b a m}$ & $h_{c c r-c o r r}-h_{c c r \_b a m}$ \\
\hline mean & 0.0032 & 0.0038 & -0.0001 \\
\hline std & 0.0152 & 0.0164 & 0.0018 \\
\hline count & 74 & 58 & 58 \\
\hline t-stat & 1.8087 & 1.7788 & -0.4114 \\
\hline signif lev & $10 \%$ & $10 \%$ & \\
\hline \multicolumn{4}{|c|}{ Panel B. JY } \\
\hline & $h_{c c r-d c c}-h_{c c r-c o r r}$ & $h_{c c r-d c c}-h_{c c r-b a m}$ & $h_{c c r \_c o r r}-h_{c c r \_b a m}$ \\
\hline mean & -0.0005 & 0 & 0.0008 \\
\hline std & 0.0037 & 0.0028 & 0.0035 \\
\hline count & 78 & 62 & 62 \\
\hline t-stat & -1.1004 & 0.1176 & 1.6953 \\
\hline signif lev & & & $10 \%$ \\
\hline \multicolumn{4}{|c|}{ Panel C. Gold } \\
\hline & $h_{c c r-d c c}-h_{c c r-c o r r}$ & $h_{c c r-d c c}-h_{c c r \_b a m}$ & $h_{c c r-c o r r}-h_{c c r-b a m}$ \\
\hline mean & 0.0061 & 0.0064 & 0.0001 \\
\hline std & 0.0261 & 0.0254 & 0.0087 \\
\hline count & 153 & 129 & 129 \\
\hline t-stat & 2.8874 & 2.8625 & 0.162 \\
\hline signif lev & $1 \%$ & $1 \%$ & \\
\hline \multicolumn{4}{|c|}{ Panel D. HE Differences Aggregated across Assets } \\
\hline & $h_{c c r-d c c}-h_{c c r-c o r r}$ & $h_{c c r-d c c}-h_{c c r-b a m}$ & $h_{c c r-c o r r}-h_{c c r \_b a m}$ \\
\hline mean & 0.0033 & 0.0036 & -0.0004 \\
\hline std & 0.0137 & 0.0141 & 0.0039 \\
\hline count & 222 & 174 & 174 \\
\hline t-stat & 3.5345 & 3.3777 & -1.5283 \\
\hline signif lev & $1 \%$ & $1 \%$ & \\
\hline
\end{tabular}

Table 5 repeats the analyses of Table 4 except that it analyzes the first and second halves separately to see if the HE difference results are stable across halves. Though the sample sizes in each half are not large, the HE is generally higher for $h_{c c r} d c c$ than for the other bias-adjustment approaches across assets and halves (the JY is an exception). While the HE aggregation across assets for the 1st half is not statistically significant, it is significant at the $1 \%$ level for the 2 nd half. 
Table 5. Stability of the HE Differences across $h_{c c r}$ Bias-Adjustment Methods.

\begin{tabular}{|c|c|c|c|}
\hline \multicolumn{4}{|c|}{ Panel A. S\&P500 1st Half } \\
\hline & $h_{c c r-d c c}-h_{c c r \_c o r r}$ & $h_{c c r-d c c}-h_{c c r \_b a m}$ & $h_{c c r-c o r r}-h_{c c r-b a m}$ \\
\hline mean & 0.0004 & 0.0007 & 0.0002 \\
\hline std & 0.0025 & 0.0033 & 0.0016 \\
\hline count & 37 & 21 & 21 \\
\hline t-stat & 1.0708 & 0.9055 & 0.7225 \\
\hline \multicolumn{4}{|l|}{ signif lev } \\
\hline \multicolumn{4}{|c|}{ S\&P500 2nd Half } \\
\hline mean & 0.0059 & 0.0056 & -0.0003 \\
\hline std & 0.0211 & 0.0203 & 0.0019 \\
\hline count & 37 & 37 & 37 \\
\hline t-stat & 1.7108 & 1.6902 & -0.9254 \\
\hline signif lev & $10 \%$ & $10 \%$ & \\
\hline \multicolumn{4}{|c|}{ Panel B. JY 1st Half } \\
\hline mean & -0.0008 & -0.0004 & 0.0014 \\
\hline std & 0.0049 & 0.0039 & 0.0053 \\
\hline count & 39 & 23 & 23 \\
\hline t-stat & -1.0677 & -0.5421 & 1.2426 \\
\hline \multicolumn{4}{|l|}{ signif lev } \\
\hline \multicolumn{4}{|c|}{ JY 2nd Half } \\
\hline mean & -0.0001 & 0.0003 & 0.0004 \\
\hline std & 0.0018 & 0.0019 & 0.0019 \\
\hline count & 39 & 39 & 39 \\
\hline t-stat & -0.2797 & 1.0794 & 1.3287 \\
\hline signif lev & & & $10 \%$ \\
\hline \multicolumn{4}{|c|}{ Panel C. Gold 1st Half } \\
\hline mean & 0.0053 & 0.0066 & 0.0011 \\
\hline std & 0.033 & 0.0346 & 0.0039 \\
\hline count & 76 & 52 & 52 \\
\hline t-stat & 1.4141 & 1.3841 & 2.1034 \\
\hline signif lev & $10 \%$ & $10 \%$ & $5 \%$ \\
\hline \multicolumn{4}{|c|}{ Gold 2nd Half } \\
\hline mean & 0.0068 & 0.0063 & -0.0006 \\
\hline std & 0.0169 & 0.0169 & 0.0107 \\
\hline count & 77 & 77 & 77 \\
\hline t-stat & 3.5346 & 3.2453 & -0.4598 \\
\hline signif lev & $1 \%$ & $1 \%$ & \\
\hline
\end{tabular}


Table 5. Cont.

\begin{tabular}{cccc}
\hline \multicolumn{4}{c}{ Panel D. Aggregated across Assets 1st Half } \\
\hline & $h_{c c r-d c c}-h_{c c r-c o r r}$ & $h_{c c r-d c c}-h_{c c r-b a m}$ & $\boldsymbol{h}_{c c r-c o r r}-h_{c c r-b a m}$ \\
\hline mean & 0.0007 & 0.0006 & -0.0003 \\
\hline std & 0.007 & 0.0071 & 0.0048 \\
\hline count & 111 & 63 & 63 \\
\hline t-stat & 1.0398 & 0.7008 & -0.5409 \\
\hline signif lev & Aggregated across Assets 2 nd Half \\
\hline mean & 0.0058 & 0.0053 & -0.0005 \\
\hline std & 0.0177 & 0.0166 & 0.0032 \\
\hline count & 111 & 111 & 111 \\
\hline t-stat & 3.4493 & 3.3615 & -1.6791 \\
\hline signif lev & $1 \%$ & $1 \%$ & $10 \%$ \\
\hline
\end{tabular}

\section{Conclusions}

The goal of futures hedging is to reduce the firm's risk and increase its value. A key consideration for a futures hedger is the ratio of futures assets to short over the number of spot assets long, i.e., the futures hedge ratio.

This paper proposes new dynamic conditional futures hedge ratios. It studies: (1) the economics-based carry-cost rate hedge ratio introduced in Leistikow et al. (2020) and employed in Leistikow and Chen (2019), (2) a hedge ratio based on the Engle (2002) statistics-based dynamic conditional correlation model, and (3) three bias-adjusted versions of the carry-cost rate hedge ratio, where one uses the Engle (2002) dynamic conditional correlation model to adjust the bias. The hedge ratios' values and hedging performances are compared to those of two common benchmark hedge ratios (the traditional and "naive" hedge ratios) across three broad asset classes, two sub-periods, and three rolling window sizes.

The newly proposed economics-based carry-cost rate hedge ratio augmented with the Engle (2002) dynamic conditional correlation between the spot and futures prices nearly always (1) provides the highest hedging effectiveness and (2) has a statistically significantly higher hedging effectiveness than that of the other hedge ratio approaches across assets, sub-periods, and rolling window sizes.

All of the bias-adjusted carry-cost rate hedge ratios provide statistically significantly superior hedging performance across assets, sub-periods, and rolling window sizes to that of the "naive" hedge ratio of $1, h_{1}$, advocated by Wang et al. (2015). However, this should not be interpreted as a general result. The poor hedging performance of $h_{1}$ is expected when the upward biased carry-cost rate hedge ratio, $h_{c c r}$, is significantly less than 1 as it was for these assets and time periods. Nevertheless, the carry-cost rate varies across assets, currency denominations, and time; thus, in the rare instances that the upward biased $h_{c c r}$ is above 1 by its bias, the risk minimizing hedge ratio would also be $h_{1}$.

Unlike $h_{1}$, the statistics-based traditional hedge ratio, though inefficient compared to $h_{c c r}$, is correlated with the changing carry-cost rate. Of the three augmented carry-cost rate hedge ratios, only the one augmented with the Engle (2002) dynamic conditional correlation between the spot and futures prices nearly always provided higher hedging performance than the traditional hedge ratio.

Overall, our findings suggest that an effective hedge-ratio model needs to consider both the economic motivation of the model parameters and the dynamic nature of the correlations between a futures contract and its underlying asset. On the other hand, we acknowledge that our time-series models are estimated using historical data, which may 
be susceptible to backward bias. In future research, more fruitful work can be done by incorporating forward-looking data (e.g., options data) as part of the information set into multivariate time-series models.

Author Contributions: Conceptualization, methodology, and writing: D.L. and Y.T., Data-gathering, analysis, and proofreading: W.Z. All authors have read and agreed to the published version of the manuscript.

Funding: This research received no external funding.

Informed Consent Statement: Not applicable.

Data Availability Statement: All data came from Bloomberg.

Conflicts of Interest: The authors declare no conflict of interest.

\section{Notes}

1 Many attribute this approach to Ederington (1979). Ferguson and Leistikow (1999) show that the $\Delta$ S term should be reduced by the period's carry-cost. Henceforth, we define $\Delta S$ as the carry-cost adjusted spot price change. Ferguson and Leistikow (1999) further shows that the carry-cost-adjusted spot price change hedge ratio calculation method is similar to, but theoretically superior to, the ECM hedge ratio advanced and studied by Kroner and Sultan (1993) and Ghosh and Clayton (1996).

2 The 1008 trading days, i.e., 4 years, rolling window size is arbitrary. For a robustness check, we replicate our analyses using 2and 6-years rolling windows. Our results remain intact. The results are available upon request.

3 This modeling has been shown to produce superior empirical performance in a variety of situations. See, e.g., Engle (2002), Chiang et al. (2007), and Baur and Lucey (2010).

$4 \quad$ For a general carry-cost rate discussion, see Brennan (1958).

5 In discrete time hedging, $T$ in Equation (11) is replaced by the hedge lift date's years to maturity, i.e., $T-t$.

6 For a more complete discussion of the advantages and disadvantages of the carry cost rate based hedge ratios, see Leistikow et al. (2020).

$7 \quad$ The goal was to get a long (at least back to 12 July 1990) series for the short term (ideally overnight for the daily data and weekly for the weekly data) US nearly riskless interest rate. Bloomberg's US 1-week Repo rate data begins on 23 July 1998 and has several gaps. Their overnight repo rate data begins at the same time as the 1-week repo rate data but is missing for about 100 more dates. Surprisingly, the 1-week rate average was about 2.5 basis points less than the overnight rate, but still this seems a minor difference. From these 2 series, we created a merged 1-week/1-day repo series: it is the 1-week repo rate unless it is unavailable, then it is the overnight repo rate -2.5 basis points. Bloomberg also has data on two 1-month interest rate series (repo rate and Libor) that go back farther than the above discussed (preferred, but unavailable) shorter (1-week/1-day) interest rates. The 1-month repo rate was about 20 basis points lower than the Libor on average. From these two 1-month rates, we created a 1 month merged rate series-it was the repo rate when available and Libor-20 basis points, otherwise. Finally, we created an overall series from the two merged series we just created (i.e., the 1-week/1-night series and the 1-month series). Since the merged 1 month series averaged 0.5 basis points less than the merged 1week/1night series, it is the merged 1 week/day repo series unless it is unavailable, in which case it is the 1 month series +0.5 basis points. This final merged series is the short term nearly riskless US interest rate used in CCR calculations for the various assets.

8 For the S\&P500 and JY, which have quarterly maturities, we lose the first 16 contracts to construct the first 1008-days rolling window. The gold contracts have bimonthly maturities, so we lose the first 24 contracts to construct the first 1008-days rolling window. Therefore, in this section, the results of the S\&P500, JY, and gold are based on 74, 78, and 153 contracts, respectively.

9 There are more statistics-based hedge ratios such as the GARCH method and its variants (e.g., Sarno and Valente (2000), Shaffer and Demaskey (2005), Alizadeh et al. (2008), Lee et al. (2009), and Wang et al. (2015). Alexander and Barbosa (2007) find the GARCH model hedging performance to be inferior to that for $h_{t}$, while Lien (2009) finds it is inferior except in small samples under special conditions.

\section{References}

Alexander, Carol, and Andreza Barbosa. 2007. Effectiveness of Minimum-Variance Hedging. Journal of Portfolio Management 33: 46-59. [CrossRef]

Alizadeh, Amir, Nikos Nomikos, and Panos Pouliasis. 2008. A Markov Regime Switching Approach for Hedging Energy Commodities. Journal of Banking \& Finance 32: 1970-83.

Bali, Turan G., and Robert F. Engle. 2010. The Intertemporal Capital Asset Pricing Model with Dynamic Conditional Correlations. Journal of Monetary Economics 57: 377-90. [CrossRef]

Bali, Turan G., Robert F. Engle, and Yi Tang. 2017. Dynamic Conditional Beta Is Alive and Well in the Cross Section of Daily Stock Returns. Management Science 63: 3531-997. [CrossRef] 
Baur, Dirk, and Brian Lucey. 2010. Is Gold a Hedge or a Safe Haven? An Analysis of Stocks, Bonds and Gold. Financial Review 45: 217-29. [CrossRef]

Brennan, M. 1958. The Supply of Storage. American Economic Review 48: 50-71.

Chiang, Thomas, Bang Jeon, and Huimin Li. 2007. Dynamic Correlation Analysis of Financial Contagion: Evidence from Asian Markets. Journal of International Money and Finance 26: 1206-28. [CrossRef]

Ederington, Louis. 1979. The Hedging Performance of the New Futures Markets. Journal of Finance 34: 157-70. [CrossRef]

Engle, Robert F. 2002. Dynamic Conditional Correlation: A Simple Class of Multivariate Generalized Autoregressive Conditional Heteroskedasticity Models. Journal of Business and Economic Statistics 20: 339-50. [CrossRef]

Ferguson, Robert, and Dean Leistikow. 1999. Futures Hedge Profit Measurement, Error-Correction Model vs. Regression Approach Hedge Ratios, and Data Error Effects. Financial Management 28: 118-25. [CrossRef]

Ghosh, Asim, and Ronnie Clayton. 1996. Hedging with International Stock Index Futures: An Intertemporal Error Correction Model. Journal of Financial Research 19: 477-91. [CrossRef]

Hull, John. 2018. Options, Futures and Other Derivatives, 10th ed. London: Pearson Education Inc.

Kroner, Kenneth, and Jahangir Sultan. 1993. Time-Varying Distributions and Dynamic Hedging with Foreign Currency Futures. Journal of Financial and Quantitative Analysis 28: 535-51. [CrossRef]

Lee, Cheng-Few, Kehluh Wang, and Yan Long Chen. 2009. Hedging and Optimal Hedge Ratios for International Index Futures Markets. Review of Pacific Basin Financial Markets and Policies 12: 593-610. [CrossRef]

Leistikow, Dean, Ren-Raw Chen, and Yuewu Xu. 2020. Spot Asset Carry Cost Rates and Futures Hedge Ratios. Working Paper. Bronx: Gabelli School of Business, Fordham University, forthcoming in Review of Quantitative Finance and Accounting.

Leistikow, Dean, and Ren-Raw Chen. 2019. Carry Cost Rate Regimes and Futures Hedge Ratio Variation. Journal of Risk and Financial Management 12: 78. [CrossRef]

Lien, Donald. 2009. Note on the Hedging Effectiveness of GARCH Models. International Review of Economics and Finance 18: 110-12. [CrossRef]

Sarno, Lucio, and Giorgio Valente. 2000. The Cost of Carry Model and Regime Shifts in Stock Index Futures Markets: An Empirical Investigation. The Journal of Futures Markets 20: 603-24. [CrossRef]

Shaffer, David, and Andrea Demaskey. 2005. Currency Hedging Using the Mean-Gini Framework. Review of Quantitative Finance and Accounting 25: 125-37. [CrossRef]

Wang, Yidong, Chongfeng Wu, and Li Yang. 2015. Hedging with Futures: Does Anything Beat the Naïve Hedging Strategy? Management Science 61: 2870-89. [CrossRef] 